\title{
Comparative Pre-clinical Efficacy of Chinese and Indian Cultivars of Bitter Melon (Momordica charantia) against Pancreatic Cancer
}

\author{
Kushal Kandhari ${ }^{1}$, Sandeep Paudel ${ }^{1}$, Komal Raina ${ }^{1,2}$, Chapla Agarwal', Rama Kant', Michael F. Wempe ${ }^{1,3}$, \\ Cindy O'Bryant ${ }^{1,3}$, Rajesh Agarwal ${ }^{1,3}$ \\ 'Department of Pharmaceutical Sciences, Skaggs School of Pharmacy and Pharmaceutical Sciences, University of \\ Colorado - Anschutz Medical Campus, Aurora, CO, ${ }^{2}$ Department of Pharmaceutical Sciences, South Dakota State \\ University, Brookings, SD, ${ }^{3}$ University of Colorado Cancer Center, University of Colorado - Anschutz Medical Campus, \\ Aurora, CO, USA
}

Given the high rates of incidence and mortality associated with pancreatic cancer (PanC), there is a need to develop alternative strategies to target PanC. Recent studies have demonstrated that fruits of bitter melon (Momordica charantia) exhibit strong anticancer efficacy against PanC. However, the comparative effects of different bitter melon varieties have not been investigated. This has important implications, given that several bitter melon cultivars are geographically available but their differential effects are not known; and that on a global level, individuals could consume different bitter melon varieties sourced from different cultivars for anti-PanC benefits. Considering these shortcomings, in the present study, comparative pre-clinical anti-PanC studies have been conducted using lyophilized-juice and aqueous-methanolic extracts of the two most widely consumed but geographically diverse bitter melon varieties (Chinese [bitter melon juice; BMJ] and Indian [bitter melon extract; BME] variants). We observed that both BMJ and BME possess comparable efficacy against PanC growth and progression; specifically, these preparations have the potential to (a) inhibit PanC cell proliferation and induce cell death; (b) suppress PanC tumor growth, proliferation, and induce apoptosis; (c) restrict capillary tube formation by human umbilical vein endothelial cells, and decrease angiogenesis in PanC tumor xenografts. Thus, given the comparable pre-clinical anti-PanC efficacy of bitter melon cultivars, the geographical non-availability of a certain cultivar should not be a limiting factor in selecting a variant for moving forward for future clinical use/clinical trials either as a preventive or a therapeutic alternative for targeting PanC.

Key Words Pancreatic neoplasms, Bitter melon, Apoptosis, Angiogenesis, Natural/dietary agents

\section{INTRODUCTION}

Pancreatic cancer (PanC) has proven to be one of the most fatal malignancies known to humankind in the past few decades [1]. With the lowest 5-year survival rate of $\sim 10 \%$ among all cancers, it accounts for $\sim 3 \%$ of all cancer incidence and $\sim 7 \%$ of all cancer deaths [2]; it has the highest mortality rate among all primary cancers. According to the American Cancer Society, in 2021, 60,000 Americans would be diagnosed with PanC, and there would be $\sim 48,000$ deaths due to this malignancy [2]. The aggressive nature of PanC can be attributed to the poor prognosis and the asymptomatic and brisk progression to advanced stages [1]. Multiple risk factors are associated with PanC such as obesity, diabetes, chronic pancreatitis, cigarette smoking, family history, and alcohol consumption [3]. The primary curative option of surgical resection is precluded in most patients of PanC because of its tricky location and clinical diagnosis in advanced stages [1]. Apart from surgery, chemotherapy is another option for treating PanC. However, recent research has shown that PanC presents an exceptional resistance to available chemotherapeutic drugs such as gemcitabine. Also, treatment regimens involving chemotherapeutic drugs are often associated with many side effects and poor overall survival [4-6]. Therefore, there is an imminent need to establish better strategies that overcome drug resistance and target the molecular pathways

Received December 9, 2021, Revised December 21, 2021, Accepted December 21, 2021

Correspondence to Rajesh Agarwal, E-mail: Rajesh.Agarwal@cuanschutz.edu, https://orcid.org/0000-0002-9455-0235

Check for updates 
driving PanC progression with minimal toxicity to the normal cells.

In this regard, over the past few decades, several research groups have focused on elucidating the potential of natural dietary/non-dietary products as anti-cancer agents with minimal toxicity to healthy cells [7]. Many of these natural products have shown remarkable anti-cancer potential in cancers of different origins, including PanC $[6,8]$. These products have been reported to exert anti-cancer efficacy via various mechanisms such as scavenging free radicals, suppressing growth and proliferation of malignant cells, and modulating expression levels of downstream oncogenes, tumor-suppressor genes, kinases, transcriptional factors, cyclins, caspases, and cancer stem cells [9-11].

Accordingly, in recent years, our laboratory has focused on investigating the efficacy of bitter melon juice (BMJ) on the preventive intervention of PanC [5,12-14]. Bitter melon fruit (Momordica charantia) is a vegetable widely consumed in Asia, Africa, and South America [15]. Bitter melon has been known to have several uses beyond the culinary realm as it has been widely used as a folk medicine, primarily as a remedy for diabetes [16]. BMJ/extracts have been studied for their anti-bacterial, anti-inflammatory, antioxidant, anti-viral, immunomodulatory, and anti-cancer activities [12,17]. Our published research has shown that BMJ exhibits strong anti-cancer efficacy against PanC in in vitro and tumor xenograft models $[5,14]$. We have also demonstrated that BMJ could reverse gemcitabine resistance in PanC patient-derived xenograft tumors $[5,13]$. The major limitations of the above studies, however, are that the comparative effects of different bitter melon varieties have not been investigated. This has important implications, given that several bitter melon cultivars are geographically available but their differential effects are not known, and that on a global level, individuals could consume different bitter melon varieties sourced from different cultivars for anti-PanC benefits. Considering these shortcomings, the present pilot study aims to address this lacuna in the current literature; thus, comparative pre-clinical anti-PanC studies have been conducted using the two most widely consumed but geographically different bitter melon varieties (Chinese and Indian variants).

\section{MATERIALS AND METHODS}

\section{Cell lines and reagents}

Human pancreatic adenocarcinoma cells PANC-1 were purchased from ATCC (Manassas, VA, USA). PANC-1 cells were grown under standard culture conditions $\left(37^{\circ} \mathrm{C}, 95 \%\right.$ humidified air, and $5 \% \mathrm{CO}_{2}$ ) in RPMl-1640 medium and supplemented with 10\% FBS and 1\% Penicillin-Streptomycin from Thermo Fisher Scientific (Waltham, MA, USA). Human umbilical vein endothelial cells (HUVECs) were purchased from Lonza (Walkersville, MD, USA) and cultured in EBM-2 (endothelial cell growth basal medium 2) supplemented with endothelial cell growth medium 2 (EGM-2) Bullet kit under standard culture conditions. Antibody for Ki-67 (\#ab16667) was from Abcam (Cambridge, MA, USA). The cleaved caspase 3 (Asp175) (\#9661) antibody was from Cell Signaling Technology (Beverly, MA, USA), and the antibody for cluster of differentiation 31 (CD-31) (\#sc-376764) was purchased from Santa Cruz Biotechnology (Dallas, TX, USA). Phenol red and LDEV-free Matrigel (\#356237) were obtained from Corning Life Sciences (Corning, NY, USA), and 0.4\% trypan blue dye (\#15250061) was from Thermo Fisher Scientific.

\section{Cultivar sources of bitter melon preparations}

Chinese variety of bitter melon fruit (light green, smooth with longitudinal ridges) was sourced from a local grocery store; bitter melon was deseeded and juiced using a juicer. BMJ was centrifuged at $3,000 \times g$ for 30 minutes at $4^{\circ} \mathrm{C}$. Thereafter, BMJ was vacuum filtered using a $0.22-\mu \mathrm{m}$ filter and aliquoted in vials for batch lyophilization using a Lyostar3 lyophilizer system (SP Scientific, Ulster County, NY, USA). All the steps for BMJ preparation are well standardized and described previously [18]. Indian variety of bitter melon fruit (dark green, pebbly rough ridges) was sourced from Bio-gen Extracts Pvt. Ltd (Bangalore, India) as aqueous-methanolic extracts (bitter melon extract [BME]) of the bitter melon fruit. The BME preparations varied in their concentration of bitters $(5 \%, 10 \%$, and $15 \%$ of bitters: saponins such as Charantin and are hereafter referred to as BME-P1, BME-P2, and BME-P3, respectively. All the preparations (BMJ and BMEP1-P3) were subjected to in-house developed and standardized LC/MS-MS chemical analysis as detailed by us earlier [14].

\section{Cell growth and death assay}

PANC-1 cells were seeded for 24 hours and then treated with varying concentrations of BMJ $(2 \%$ and $4 \%$, v/v) or BME $(2 \%, 4 \%$, and $6 \%, v / v)$ concentrations. For control groups, cells were treated with dimethyl sulfoxide (DMSO) $(<0.1 \%)$. The trypan blue assay was performed after 48 and 72 hours to assess the effect of BMJ or BME on cell viability. HUVECs were seeded for 24 hours and treated with $0.5 \%$ to $2 \% \mathrm{v} /$ $\checkmark$ BMJ or BME. After 24 hours of treatment, cell viability was determined using the trypan blue dye exclusion assay on HUVEC and PANC-1 cells as described earlier [19].

\section{Endothelial cell capillary-like tube formation assay}

Phenol-red and LDEV-free Matrigel were added in a prechilled 24 well plate $(150 \mu \mathrm{L} /$ well) and allowed to polymerize for 1 hour at $37^{\circ} \mathrm{C}$. HUVECs $\left(4 \times 10^{4}\right.$ cells per well) in complete EBM-2 medium (EBM-2 Basal medium supplemented with EGM-2 Bullet kit) were simultaneously seeded with varying concentrations of BMJ or BME (concentrations non-toxic to cells: $0.25 \%$ and $0.5 \% \mathrm{v} / \mathrm{v}$ ). After 3 hours of incubation, generation/disruption of formed tubular structures was exam- 
ined as a function of time and photographed at different time intervals captured using Axiocam 105 color digital camera fitted on a Zeiss (Carl Zeiss, Oberkochen, Germany) inverted microscope. Angiogenesis analyzer plugin of ImageJ software (National Institute of Health, Bethesda, MD, USA) was used to quantify different parameters that define the degree of tube formation at 9 hours.

\section{PANC-1 tumor xenograft study}

All animal experiments were performed at the animal house facility of the University of Colorado-Anschutz Medical Campus, and the protocols were approved by the Institutional Animal Care and Use Committee (No. IACUC 0074). Female athymic nude mice [Crl:NU(NCr)-Foxn1nu] mice $(n=35)$ were purchased from Charles River Laboratories (Wilmington, MA, USA) and housed at the animal facility for a week for acclimatization. At $\sim 6$ weeks of age, about $2 \times 10^{6}$ PANC-1 cells suspended in $50 \mu \mathrm{L}$ of serum-free DMEM media, mixed with $50 \mu \mathrm{L}$ of Matrigel were injected subcutaneously into the right flank of each mouse. Mice ( $n=7$ per group) were initiated on BMJ or BME treatment (oral gavage: $200 \mathrm{mg} / \mathrm{kg}$ in 100 $\mu \mathrm{L}$ water). The BME mixture was sonicated for 5 minutes. For better dissolution, both BMJ and BME were prepared fresh daily prior to administration, and treatment was given 5 days a week, 24 hours after cell injection, and continued till 58 days. Mice in the control group $(n=7)$ were gavaged with $100 \mu \mathrm{L}$ water only. The tumor volume was first measured after 21 days following cell inoculation; afterward, tumor volumes were measured using digital calipers, and tumor volume were calculated using the formula $0.5236 L_{1}\left(L_{2}\right)^{2}$, where $L_{1}$ is the long axis and $L_{2}$ is the short axis as described previously [20]. The body weights were determined twice a week. After termination of the experiment (day 58), mice were sacrificed by $\mathrm{CO}_{2}$ asphyxiation followed by exsanguination. Tumor tissues, liver, spleen, and pancreas were harvested from each mouse. All tissues were partly flash-frozen in liquid nitrogen and partly formalin-fixed for pathological analyses.

\section{Histological examination and Immunohistochemical analyses}

Briefly, paraffin-embedded tissues were sectioned (5 $\mu \mathrm{m})$ and subjected to routine processing for $\mathrm{H} \& \mathrm{E}$ and immunohistochemistry (IHC) [21]. The histopathological examination of tissues was done to examine the effect of BMJ or BME on the tissue morphology. Regarding IHC, positively stained cells for Ki-67 and cleaved caspase 3 were quantified by counting brown-stained cells among the total number of cells at 5 randomly selected high power fields (400x). Quantification of CD-31 microvessel density was done by counting the number of CD-31 positive microvessels in at least 5 high power fields (400x) for each sample. Representative pictures were taken at $400 \times$ magnification using an Olympus DP27 color camera fitted on an Olympus BX43F microscope (Olympus, Tokyo, Japan).

\section{Statistical analyses}

Statistical analysis was done using GraphPad Prism (version 8.4, GraphPad Software Inc., San Diego, CA, USA) and SigmaStat (version 3.5, Jandel Scientific Software, San Jose, CA, USA). Statistical significance between control and treatment groups was determined using unpaired $t$-test or one way-ANOVA followed by Tukey's test for multiple comparisons. $P \leq 0.05$ was considered significant. Data were presented as mean \pm SEM.

\section{RESULTS}

\section{Comparative in vitro efficacy of Chinese and Indian cultivars of bitter melon against PANC-1 cells}

Trypan blue dye exclusion assay was used to assess the comparative effect of BMJ and BME on the viability of PANC1 cells. Results revealed that both BMJ and BME decreased the total cell number in a time and a dose-dependent manners at 48 and 72 hours. Treatment with $2 \%$ and $4 \%$ v/v BMJ decreased the total number of PANC-1 cells by $\sim 49 \%$ ( $P \leq$ $0.001)$ and $\sim 90 \%(P \leq 0.001)$, respectively at 72 hours. BMJ at $2 \%$ and $4 \% \mathrm{v} / \mathrm{v}$ concentrations also increased cell death in PANC-1 cells by $\sim 1.4$ and $\sim 13$ folds $(P \leq 0.001)$, respectively at 72 hours (Fig. $1 \mathrm{~A})$; cell death with a higher concentration $(4 \% \mathrm{v} / \mathrm{v} \mathrm{BMJ})$ was time-dependent. BME treatment also resulted in a decrease in the total number of PANC-1 cells. Specifically, BME-P1 (2\%, 4\%, and 6\% v/v) decreased the total number of PANC-1 cells by $\sim 27 \%$ ( $P \leq 0.01), \sim 54 \%$ ( $P \leq$ 0.001 ), and $\sim 79 \%(P \leq 0.001)$, respectively at 72 hours (Fig. 1B); BME-P2 (2\%, 4\%, and $6 \% \mathrm{v} / \mathrm{v})$ decreased the total cell number by $\sim 33 \%$ ( $P \leq 0.001), \sim 75 \%$ ( $P \leq 0.001)$, and $\sim 86 \%$ $(P \leq 0.001)$, respectively at 72 hours (Fig. 1C), and BME-P3 $(2 \%, 4 \%$, and $6 \% \mathrm{v} / \mathrm{v})$ decreased the total cell number by $\sim 12 \%(P \leq 0.05), \sim 27 \%(P \leq 0.01)$, and $\sim 53 \%(P \leq 0.001)$, respectively at 72 hours (Fig. 1D). However, BME preparations were not able to induce strong cell death in PANC-1 cells when compared to BMJ. BME-P1 and BME-P2 increased cell death by $\sim 2$ fold $(P \leq 0.05)$ and $\sim 2.5$-fold $(P \leq 0.01)$ at the highest concentration of $6 \%$ v/v at 72 hours, respectively; however, BME-P3 was not able to induce any significant cell death in PANC-1 cells.

\section{Comparative in vivo efficacy of Chinese and Indian cultivars of bitter melon against PANC-1 tumor xenografts}

Next, we examined the in vivo efficacy of BMJ and BME against the growth of PANC-1 tumor xenografts in athymic nude mice. Results indicated that administration of BMJ and BME through oral gavage (at Remove hyphen $=200 \mathrm{mg} /$ $\mathrm{kg}$ body weight doses) caused a considerable time-dependent inhibition of PANC-1 xenograft growth compared to the control. At the end of the experiment (day 58), the average tumor volume/mouse decreased from $\sim 1,507 \mathrm{~mm}^{3}$ in the 

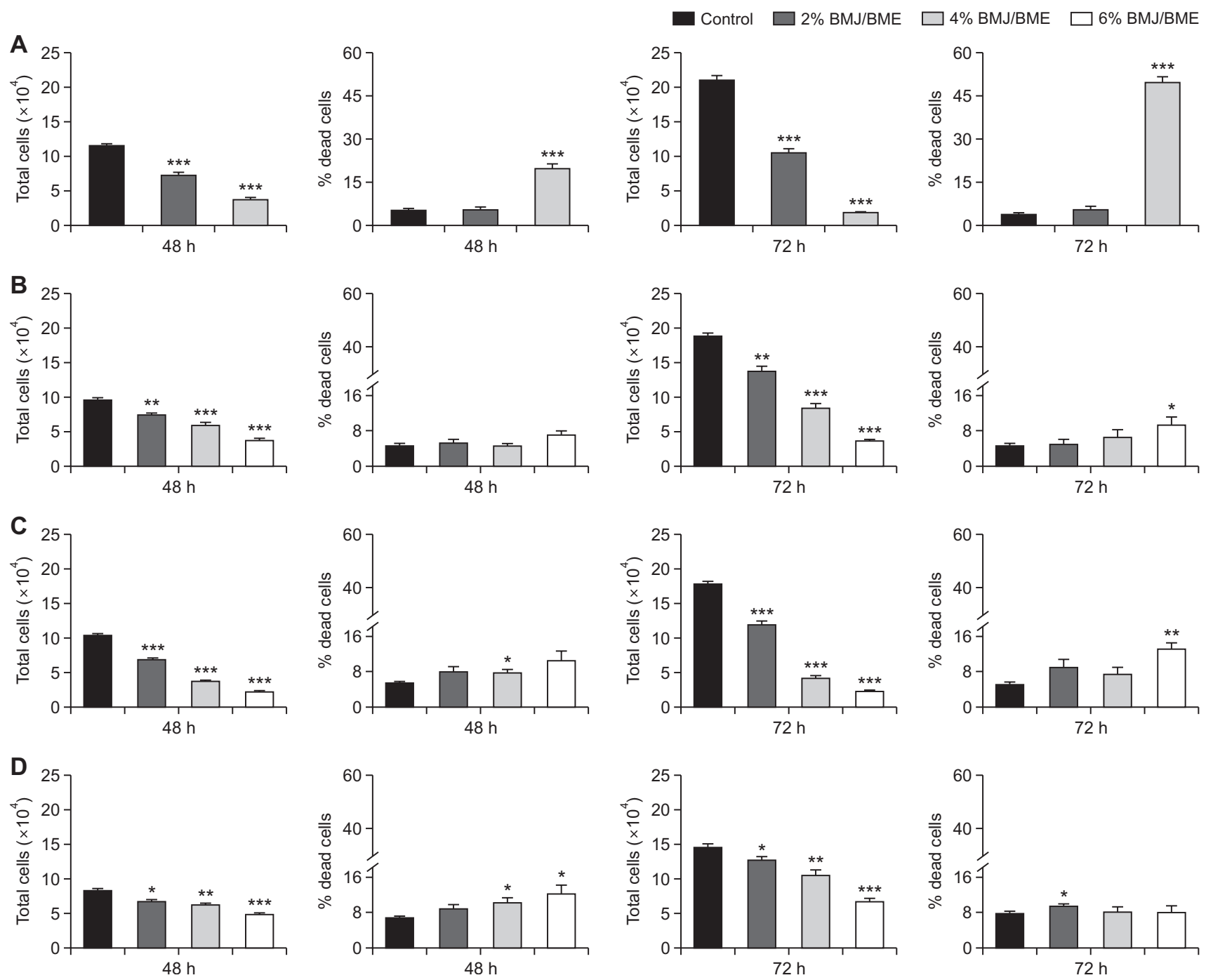

Figure 1. Treatment with bitter melon variants (BMJ and BME) decreases the cell viability of human pancreatic cancer PANC-1 cells. PANC1 cells were treated with dimethyl sulfoxide (DMSO) (control) or different concentrations of BMJ $(2 \%$ and $4 \% \mathrm{v} / \mathrm{v})$ or $\mathrm{BME}(2 \%, 4 \%$ and $6 \% \mathrm{v} / \mathrm{v})$ and processed as mentioned in the materials and methods. The trypan blue dye exclusion assay showing a decrease in total cell number and an increase in percent cell death at 48 and 72 hours by (A) BMJ, (B) BME-P1, (C) BME-P2, and (D) BME-P3. Values are means \pm SEM. BMJ, bitter melon juice; BME, bitter melon extract. ${ }^{*} P<0.05,{ }^{* \star} P<0.01,{ }^{* \star *} P<0.001$.

control group to $322 \mathrm{~mm}^{3}$ (BMJ), $478 \mathrm{~mm}^{3}$ (BME-P1), 440 $\mathrm{mm}^{3}$ (BME-P2), and $453 \mathrm{~mm}^{3}$ (BME-P2) $\mathrm{mm}^{3}$ in the treatment groups, respectively, which accounted for $\sim 79 \%$ ( $P \leq$ $0.01), \sim 68 \%(P \leq 0.05), \sim 71 \%(P \leq 0.05)$, and $\sim 70 \%(P \leq$ 0.05 ) decrease in tumor volumes, respectively (Fig. 2A). BMJ and BME treatments also reduced the tumor weights significantly when compared to the controls. Specifically, the tumor weights were decreased by $\sim 81 \%, P \leq 0.01$ (BMJ); $\sim 68 \%, P$ $\leq 0.05$ (BME-P1); $65 \%, P \leq 0.05$ (BME-P2); 71\%, $P \leq 0.01$ (BME-P3) treatment groups (Fig. 2B).

\section{Comparative anti-proliferative and apoptotic effects of Chinese and Indian cultivars of bitter melon in PanC tumors \\ Next, IHC analysis was performed to examine the compara-}

tive effect of BMJ and BME on the expression levels of proliferation (Ki-67) and apoptosis (cleaved caspase 3) markers. The results indicated a significant decrease in the proliferative index (\% positive Ki-67 cells) and an increase in the expression of cleaved caspase 3 in the treatment groups. The Ki-67 positive cells decreased by $\sim 45 \%, P \leq 0.001$ (BMJ); $\sim 22 \%, P$ $\leq 0.01$ (BME-P1); 35\%, $P \leq 0.01$ (BME-P2); 31\%, $P \leq 0.01$ (BME-P3). On the other hand, the percentage of apoptotic cells increased markedly in the treatment groups; there was a 2-4-fold increase in the expression of cleaved caspase- 3 in the BMJ and BME treatment groups compared to the control $\left({ }^{* *} P<0.01,{ }^{* \star *} P<0.001\right.$ ) (Fig. $2 \mathrm{C}, 2 \mathrm{D}$, and $2 \mathrm{E}$ ). 

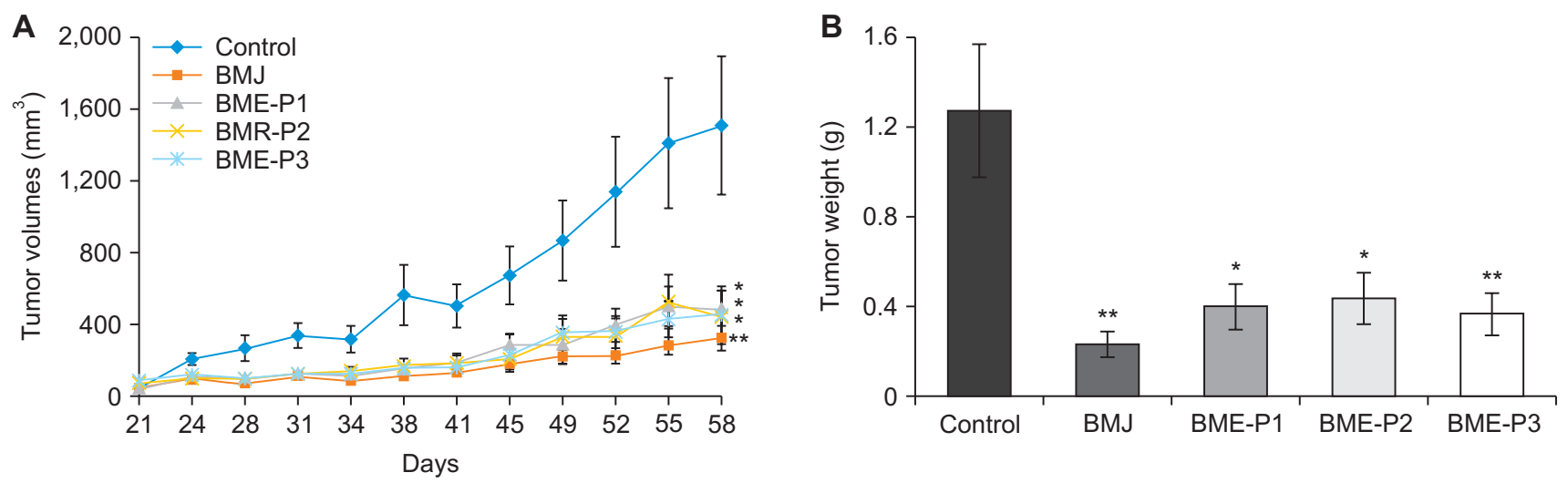

C
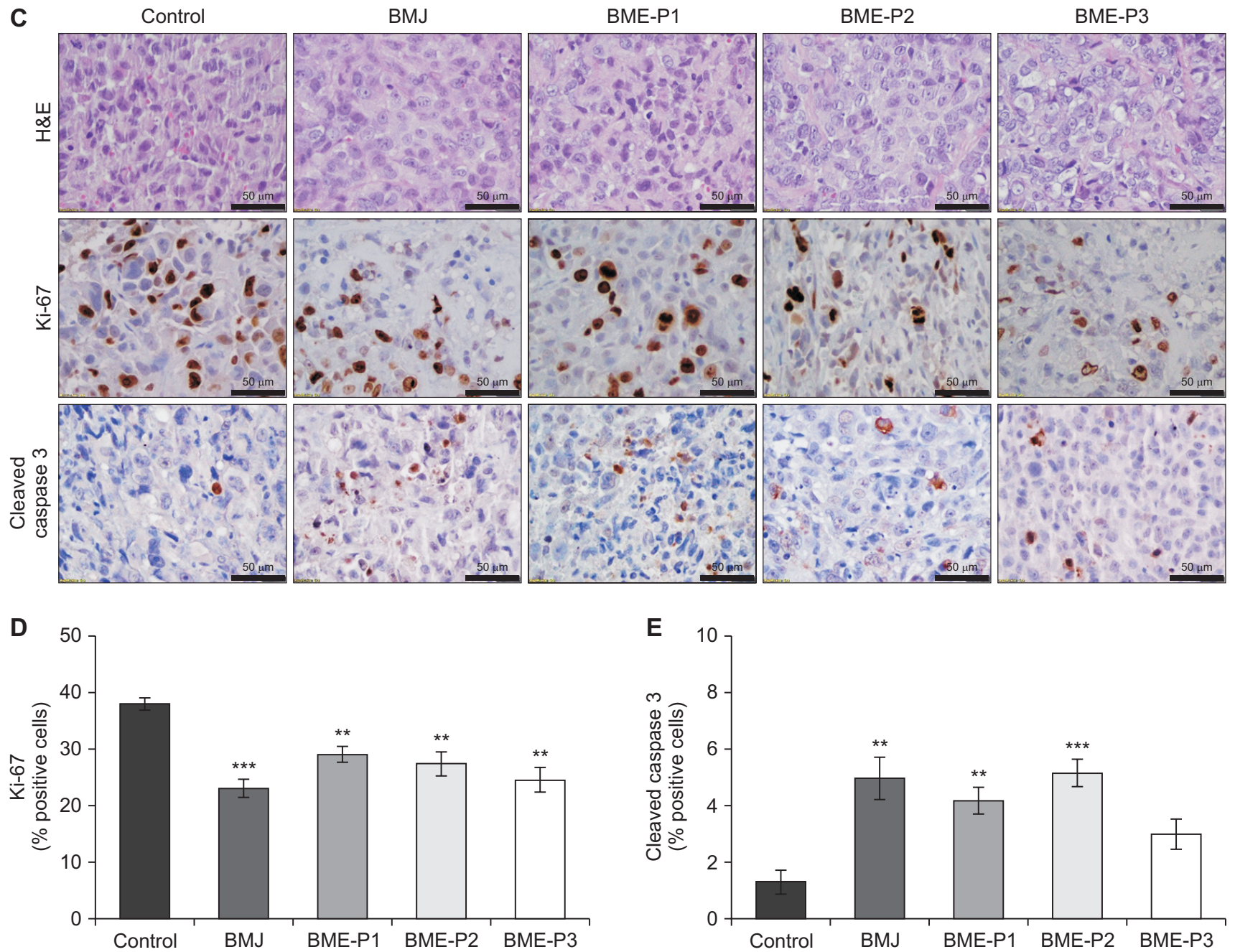

Figure 2. In vivo efficacy of bitter melon variants (BMJ and BME feeding) against human pancreatic cancer PANC-1 tumor xenografts. (A) Growth curve depicting tumor volumes plotted as a function of time. (B) Bar graph representing the tumor volumes at the end of the experiment shows a significant decrease in tumor size in the treatment groups compared to control. (C) Representative images of H\&E (tumor tissue sections) and $\mathrm{IHC}$ for Ki-67 and cleaved caspase-3. Quantitative analysis of IHC depicting the decrease in proliferation (D) and an increase in apoptosis (E) in $\mathrm{BMJ}$ and BME treated groups. For all the above experiments, mice were treated with $200 \mathrm{mg} / \mathrm{kg} \mathrm{BMJ}$ or BME in $100 \mu \mathrm{L}$ water (oral gavage). Control mice were oral gavaged with $100 \mu \mathrm{L}$ of water only as described in the material and methods section. All pictures were taken at $400 \times$ magnification. Values are means \pm SEM. BMJ, bitter melon juice; BME, bitter melon extract; IHC, Immunohistochemistry. ${ }^{*} P<0.05,{ }^{* *} P<0.01,{ }^{* * \star} P<0.001$. 


\section{Comparative non-toxic effects of Chinese and Indian cultivars of bitter melon in non-target organs in pre-clinical PanC model}

During the course of the study (as well as study end) we also investigated whether BMJ or BME causes any bodyweight loss and gross morphological/ pathological changes in non-target organs after continuous oral dosing during the study period in the mouse model used in the tumor xenograft studies. Mice from control and treatment groups were monitored during the experiment, and body weight was measured twice a week till the end of the study. Results revealed that $\mathrm{BMJ}$ and BME treatments did not induce any significant body weight changes (Fig. 3A). At the end of the study, during necropsy no abnormal organ size or gross morphological changes were observed in the organs; the liver of each animal was harvested and weighed. Results indicated that while
BMJ and BME-P1 and -P2 treatments did not affect liver weights, BMP-P3 preparation increased liver weight (Fig. $3 \mathrm{~B}$ ); however, there were no toxic effects or histopathological changes (H\&E staining) observed in the hepatic tissues (Fig. $3 C$ ). Also, no histopathological changes were observed in the spleen and pancreas tissues of BMJ and BME administered mice (Fig. 3C).

\section{Comparative anti-angiogenic effects of Chinese and Indian cultivars of bitter melon}

Next, to determine whether the anti-tumor effects of BMJ and BME were associated with differential effects of the BMJ and BME preparations on tumor angiogenesis, we performed in vitro assessments (tube-formation based angiogenesis assays) using HUVECs. Before proceeding to in vitro angiogenesis assessment assays, we first determined the non-toxic
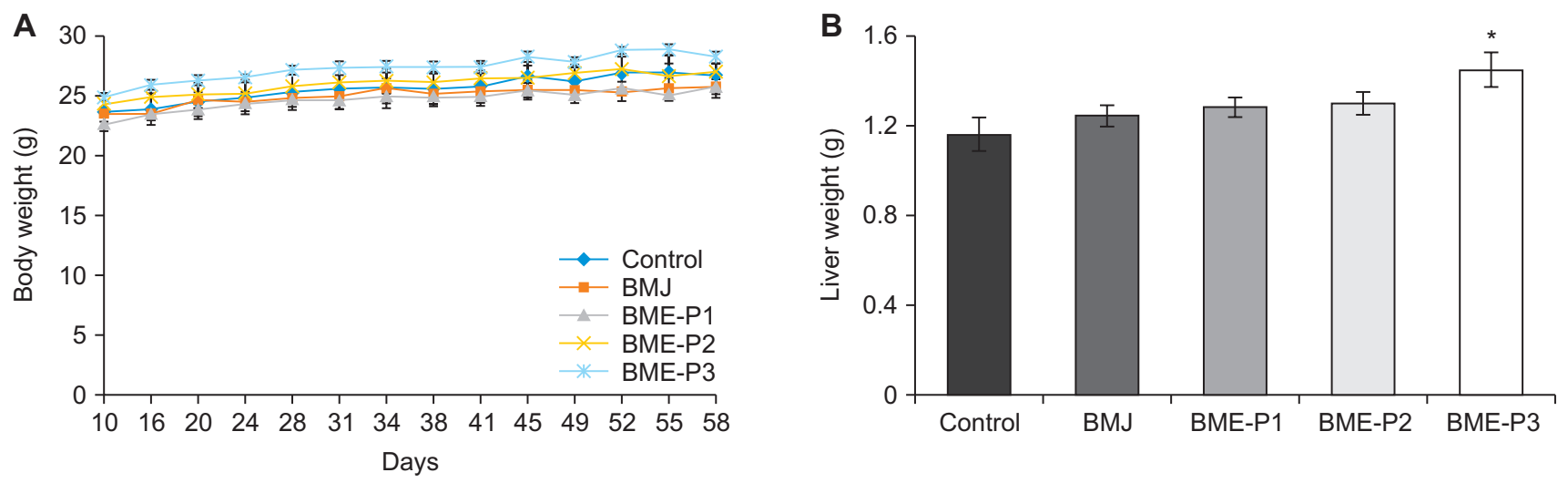

C
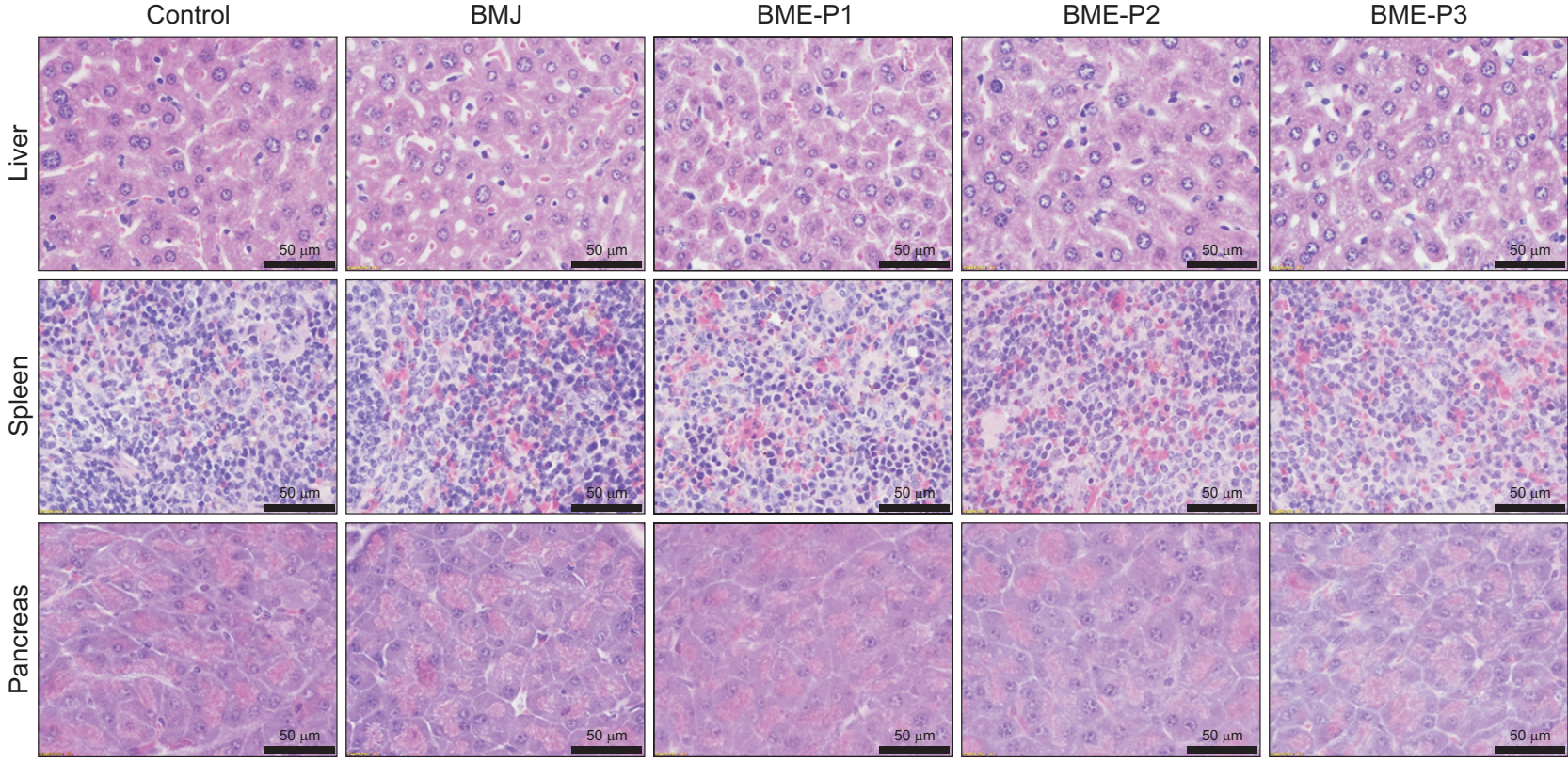

Figure 3. Non-toxic effects of bitter melon variants (BMJ and BME) in non-target organs in a pre-clinical xenograft model of pancreatic cancer. (A) Bodyweight changes as a function of time. (B) Bar graph representing the liver weight at the end of the experiment. (C) H\&E staining of non-target tissues (liver, spleen, and pancreas). All pictures were taken at $400 \times$ magnification. Values are means \pm SEM. BMJ, bitter melon juice; BME, bitter melon extract. ${ }^{*} P<0.05$. 
concentrations of BMJ and BME to ensure that the anti-angiogenic effects (if any) during the assays were not as a result of the effect on the viability of HUVECs. For this, the trypan blue assay was performed after treating the cells with BMJ and BME (at concentrations ranging from $0.5 \%$ to $2 \% \mathrm{v} / \mathrm{v}$ for 24 hours) to determine the concentration that did not affect cell growth and are least toxic to HUVEC. Results revealed that a concentration of $0.5 \% \mathrm{v} / \mathrm{v}$ of BME-P1, - $\mathrm{P} 2$, and -P3 preparations did not cause a decrease in the total cell number of $\mathrm{HU}$ VECs and were unable to induce significant cell death at 24 hours (Fig. 4). Though 0.5\% v/v BMJ did not decrease total HUVEC numbers, there was an increase in cell death at this concentration; on repeat evaluation at a lower concentration of $0.25 \% \mathrm{v} / \mathrm{v}$, BMJ did not cause cell death (data not shown). As such a concentration of $0.25 \%$ and $0.5 \% \mathrm{v} / \mathrm{v}$ of BMJ and BME preparations was used in the tube formation assays. The experiment was carried out for 24 hours to examine the effect of bitter melon on the HUVEC tube structure formation. Tubular structures were photographed at different time points ranging from 3 to 24 hours after seeding (Figure S1). We selected early time points for these studies, where we observed the least cytotoxicity of bitter melon towards HUVEC. Therefore, after 9 hours of BMJ/ BME treatments, photographs were quantified. As shown in Fig. 5A, both BMJ and BME were able to disrupt the HUVEC capillary tube formation on Matrigel. We also measured six different parameters that define capillary network formation using an angiogenesis analyzer from ImageJ. As shown in the bar diagram (Fig. 5B), the numbers of nodes, junctions, and segments, total segment length, total mesh area, and total tube length significantly decreased with BMJ and BME treatments at a concentration of $0.25 \% \mathrm{v} / \mathrm{v}\left({ }^{*} P<0.05,{ }^{* *} P<0.01,{ }^{* *} P<0.001\right)$. The antiangiogenic effect was more pronounced in the BME treated groups when compared to BMJ treated group. Specifically, the nodes and junctions were decreased by $\sim 57 \%(P \leq 0.01)$ by BMJ, but by $\sim 72 \%$ to $80 \%$ ( $P \leq 0.001)$ by BME-P1 to P3. Similarly, the segments were decreased by $\sim 61 \%(P \leq 0.01)$ by BMJ, but by $\sim 72 \%$ to $83 \%$ ( $P \leq 0.01$ ) by BME-P1 to P3. The decrease in all other parameters mentioned above was also more prominent in BME-treated cells in comparison to BMJ-treated cells suggesting a better anti-angiogenic potential of BME.

To corroborate the in vitro anti-angiogenic results, we performed IHC for PECAM-1 (CD-31, a marker for angiogenesis-microvessel formation) in the tumor tissues from the PANC-1 xenograft study. Results showed that in the tumor tissues from BMJ and BME-fed mice, there was a marked decrease in CD-31 positive scoring. Specifically, microvessel density was decreased by $\sim 25 \%, P \leq 0.05$ (BMJ); $\sim 54 \%, P$ $\leq 0.001$ (BME-P1); $60 \%, P \leq 0.001$ (BME-P2); $60 \%, P \leq$ 0.001 (BME-P3). Overall, the in vivo data also suggested that BME preparations were more effective in decreasing angiogenesis compared to BMJ (though there was no difference between the inhibitory effect of different BME preparations).

\section{DISCUSSION}

PanC is an aggressive cancer with a dismal 5-year survival rate. Apart from that, it has the highest mortality rate among all cancers [4]. Chemotherapeutic drugs, such as gemcitabine, have shown to be effective against PanC, but tumor cells readily develop resistance against such conventional cy-
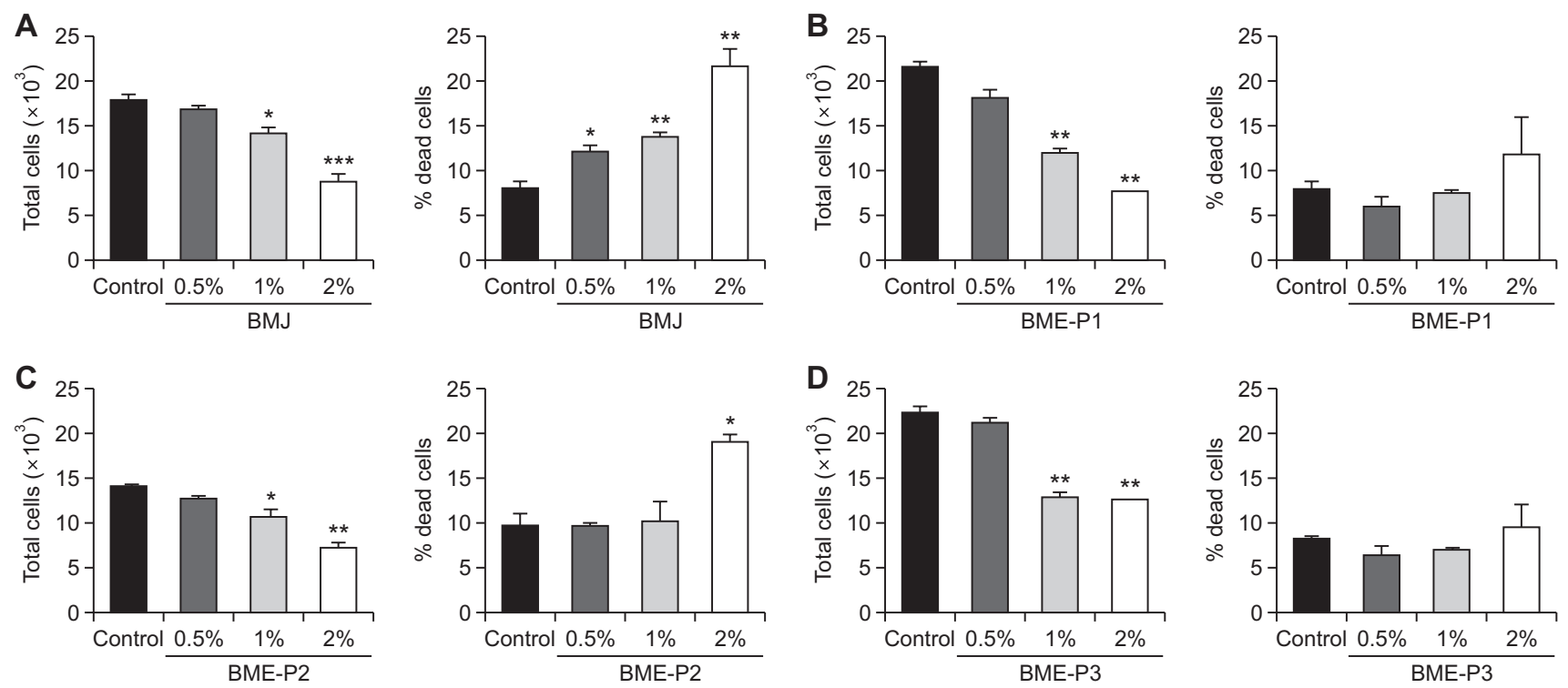

Figure 4. Effect of bitter melon variants (BMJ and BME) on the viability of human umbilical vein epithelial cells (HUVECs). HUVECs were treated with dimethyl sulfoxide (DMSO) or different concentrations of BMJ or BME $(0.5 \%$ to $2 \% \mathrm{v} / \mathrm{v})$ and processed as mentioned in the materials and methods. The trypan blue dye exclusion assay at 24 hours with (A) BMJ, (B) BME-P1, (C) BME-P2, and (D) BME-P3. Values are means \pm SEM. BMJ, bitter melon juice; BME, bitter melon extract. ${ }^{*} P<0.05,{ }^{* *} P<0.01,{ }^{* * *} P<0.001$. 
A

HUVEC
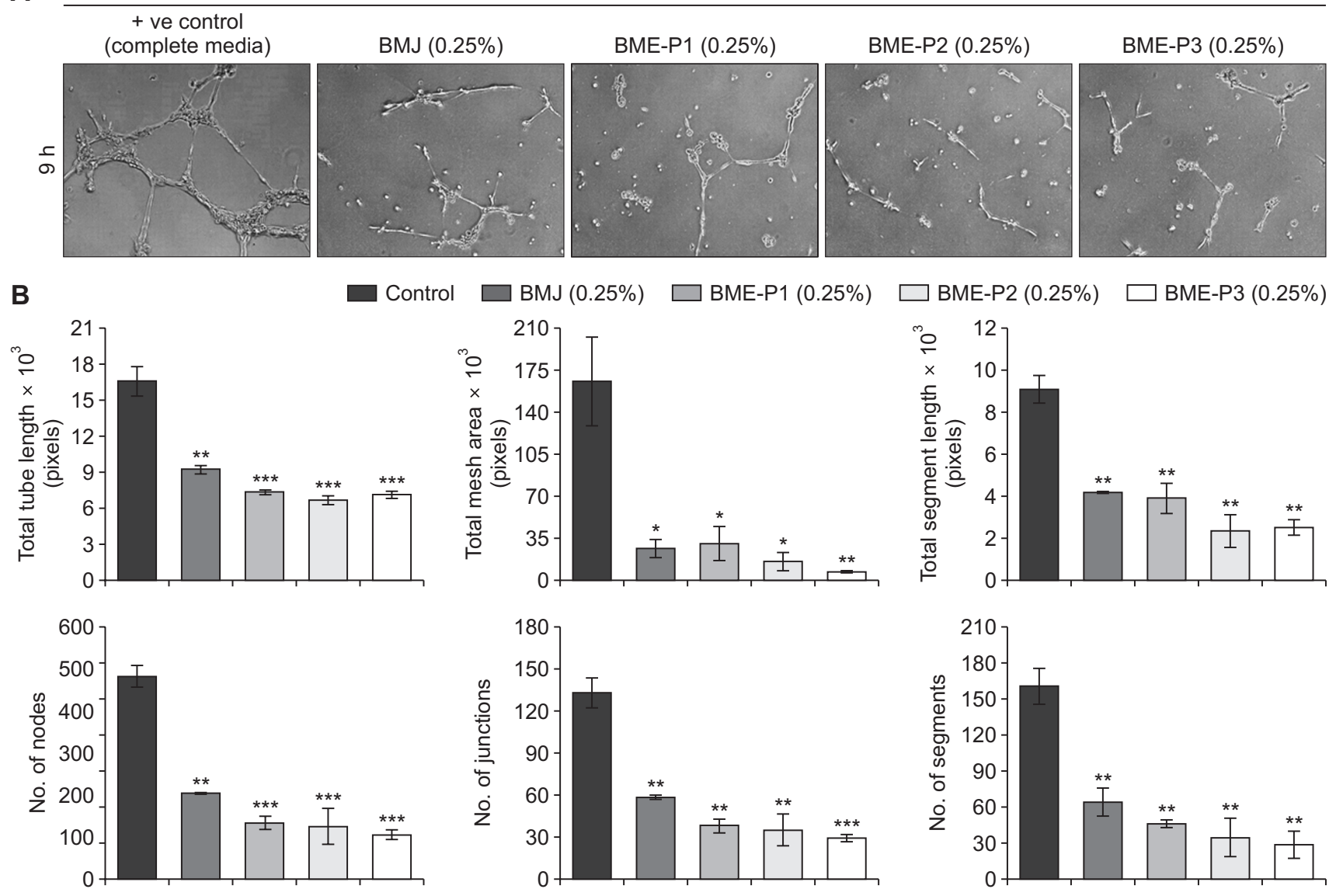

C

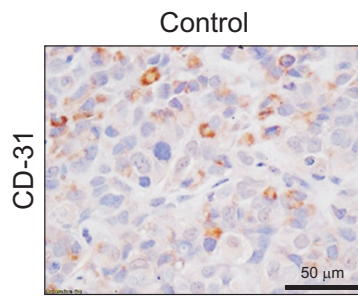

BMJ

BME-P1

BME-P2

BME-P3

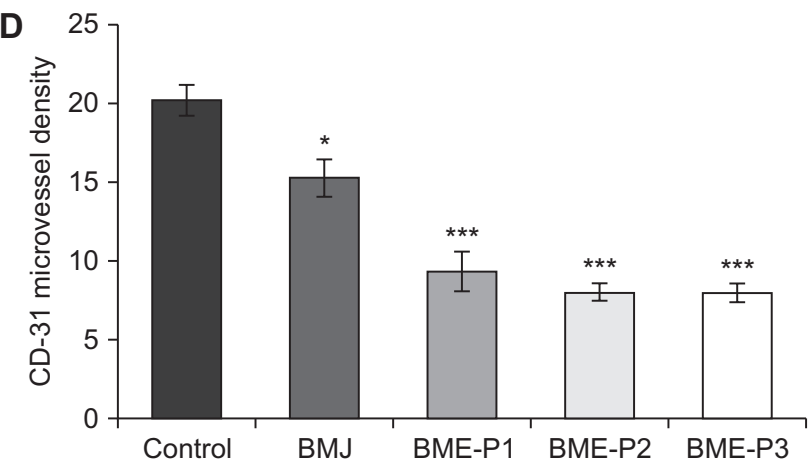

Figure 5. Anti-angiogenic effects of bitter melon variants (BMJ and BME). (A) Representative pictures demonstrate the in vitro tube formation on Matrigel at 9 hours; the formation of human umbilical vein epithelial cells (HUVECs) capillary-like structures in was inhibited by treatment with BMJ or BME (magnification: $\times 100$ ). (B) Quantitative analysis of the capillary-like tube formation assay depicting total tube length, total mesh area, and total segment length (upper panel). Also shown are bar graphs for the number of nodes, number of junctions, and number of segments (lower panel) (magnification: $\times 400$ ). (C) Representative pictures and qualitative analysis for immunohistochemical analysis of an angiogenic marker (CD-31) in PanC tumor xenografts. Values are means \pm SEM. BMJ, bitter melon juice; BME, bitter melon extract; CD-31, cluster of differentiation 31 . ${ }^{*} P<0.05$, ${ }^{* *} P<0.01,{ }^{* * *} P<0.001$. 
totoxic therapies. This highlights the critical need to develop more effective non-toxic strategies against PanC. Recently, $\mathrm{WHO}$ reported that $80 \%$ of the world's population still relies on traditional and folk medicine [17]. Epidemiological studies have shown that increased consumption of vegetables and fruits is directly linked to lower cancer cases [22]. Fruits and vegetables contain a plethora of non-nutritional chemicals with beneficial effects known as phytochemicals; these beneficial effects include antioxidant, anti-inflammatory, anti-atherosclerotic, lipid-lowering, immunomodulatory, and anti-cancer properties [23,24].

Over the past few years, increasing scientific evidence has been shown that bitter melon has shown remarkable potential as an anti-cancer agent in several cancer models [12,17]. We have shown earlier that BMJ increased the AMP-activated kinase (AMPK) phosphorylation and caused apoptotic cell death in PanC cells [13]. We also demonstrated that BMJ targets glucose metabolism, lactate flux, and cancer stem cells to exert anti-cancer efficacy in PanC cells and xenografts $[14,20]$. Although a lot of progresses were made in elucidating the underlying mechanism by which BMJ exerts its anti-cancer activity, in the present study, we attempted to investigate the comparative anti-cancer efficacy of different cultivars of bitter melon. Bitter melon fruit juice and aqueous-methanolic extracts from the Chinese (BMJ) and Indian cultivars (BME) were used in the study to determine their anti-cancer efficacy in both in vitro and xenograft tumor assays.

Cancer is defined as a disease with uncontrolled cell division, and the drug that halts this property potentially possess anti-cancer activity [25]. Our findings indicated that both BMJ and BME preparations restricted cell growth and caused apoptotic death of PANC-1 PanC cells. Interestingly, 4\% v/v of BMJ (Chinese cultivar) induced significantly higher PANC1 cell death compared to the higher concentrations of the Indian cultivar.

Further, our study demonstrated that both Chinese and Indian bitter melon preparations caused a significant decrease in PANC-1 tumor volume and weight with almost equivalent anti-PanC efficacy. Importantly, it was noted that varying concentrations of bitters $(5 \%, 10 \%$, and $15 \%$ of bitters: saponins such as Charantin) had no differential impact on the anti-cancer efficacy.

Since limitless proliferation and evasion of apoptosis are two of the several hallmarks of cancer [26], therefore, IHC analysis was performed to assess the effect of BMJ/BME in modulating the expression of Ki-67 (proliferation marker) and cleaved caspase 3 (apoptotic marker). Results revealed that both cultivars of bitter melon (BMJ and BME) decreased $\mathrm{Ki}-67$ staining and/or increased the percentage of cleaved caspase-3 positive cells. Additionally, both cultivars of bitter melon showed a comparable good safety profile ( no toxic effect on non-target organs), which is in concurrence with previously published data in human subjects [27-29].

The tumor microenvironment is characterized by complex interaction between the malignant and non-malignant cellular and extracellular components; of these, the endothelial cells are recognized as one of those critical elements in the tumor microenvironment. Pathological angiogenesis is a hallmark of cancer, and endothelial cells present in the tumor microenvironment are required for vascular tumor growth [30]. Thus, endothelial cells play a vital role in cancer progression by controlling angiogenesis. Therefore, targeting endothelial cells could restrict pathological angiogenesis and be essential in preventing and treating cancers.

Our results demonstrated that both BMJ and BME preparations have the potential to inhibit tube formation of HUVEC cells (inhibited the capillary tube formation); the anti-angiogenic effects were also evident (microvessel density decreased significantly) in tumors of BMJ- and BME-fed mice. Notably, BME preparations showed a better anti-angiogenic potential when compared to BMJ. Overall, our findings suggested that both BMJ (Chinese cultivar) and BME (Indian cultivar) exhibit promising anti-cancer and anti-angiogenic efficacy against the PanC. However, further studies are needed to understand why there is a difference between the efficacy of BMJ and BME in PanC, and that if the difference between the functional components of the two cultivars is playing a role in exhibiting different anti-cancer efficacy.

In conclusion, the central findings of this study demonstrate that bitter melon preparations from geographically distinct cultivars of bitter melon, the most commonly consumed Chinese variant (BMJ) and the Indian variant (BME), possess

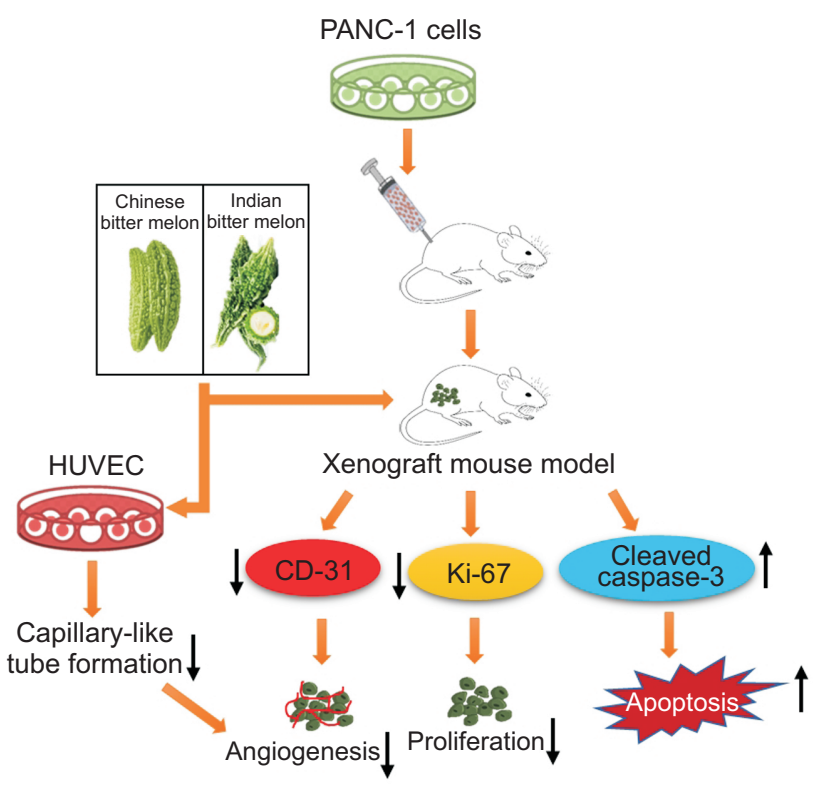

Figure 6. Comparative anticancer efficacy of bitter melon variants (BMJ and BME) in pancreatic cancer. Bitter melon restricts cell growth and induces cell death in PANC-1 cells. Bitter melon decreases tumor cell proliferation, induces apoptosis, and inhibits angiogenesis. BMJ, bitter melon juice; BME, bitter melon extract. 
comparable efficacy against PanC growth and progression. Specifically, these preparations have the potential: (a) to inhibit PanC cell proliferation and to induce cell death; (b) to suppress PanC tumor growth and proliferation, and to induce apoptosis; (c) to restrict capillary tube formation in HUVECs, and decrease angiogenesis in PanC tumor xenografts (Fig. 6). Thus, given the comparable pre-clinical anti-PanC efficacy of bitter melon cultivars, the geographical non-availability of a certain cultivar should not be a limiting factor in selecting a variant for moving forward for future clinical use/ clinical trials either as a preventive or a therapeutic alternative for targeting PanC.

\section{ACKNOWLEDGMENTS}

This work was supported by the National Institutes of Health/ National Cancer Institute grant R01CA195708 (to RA), the National Institutes of Health Office of Dietary Supplements supplemental funds R01CA195708-02S1 (to RA), and the pilot funding from the office of the Associate Dean for Research and Graduate Education (ADR), School of Pharmacy, UC AMC (to CO, RA \& MFW).

\section{CONFLICTS OF INTEREST}

No potential conflicts of interest were disclosed.

\section{SUPPLEMENTARY MATERIALS}

Supplementary materials can be found via https://doi. org/10.15430/JCP.2021.26.4.266.

\section{ORCID}

Kushal Kandhari, https://orcid.org/0000-0002-8323-7278

Sandeep Paudel, https://orcid.org/0000-0002-6389-9485

Komal Raina, https://orcid.org/0000-0003-0371-1070

Chapla Agarwal, https://orcid.org/0000-0003-1146-4890

Rama Kant, https://orcid.org/0000-0002-0550-1467

Michael F. Wempe, https://orcid.org/0000-0002-8157-5281

Cindy O’Bryant, https://orcid.org/0000-0001-6337-2221

Rajesh Agarwal, https://orcid.org/0000-0002-9455-0235

\section{REFERENCES}

1. Oberstein PE, Olive KP. Pancreatic cancer: why is it so hard to treat? Therap Adv Gastroenterol 2013;6:321-37.

2. Siegel RL, Miller KD, Fuchs HE, Jemal A. Cancer statistics, 2021. CA Cancer J Clin 2021;71:7-33.

3. Yadav D, Lowenfels $A B$. The epidemiology of pancreatitis and pancreatic cancer. Gastroenterology 2013;144:1252-61.

4. Kleeff J, Korc M, Apte M, La Vecchia C, Johnson CD, Biankin AV, et al. Pancreatic cancer. Nat Rev Dis Primers 2016;2:16022.

5. Dhar D, Raina K, Kumar D, Wempe MF, Bagby SM, Pitts TM, et al. Bitter melon juice intake with gemcitabine intervention circumvents resistance to gemcitabine in pancreatic patientderived xenograft tumors. Mol Carcinog 2020;59:1227-40.

6. Dhar D, Raina K, Agarwal R. Mechanisms and drug targets for pancreatic cancer chemoprevention. Curr Med Chem 2018;25:2545-65.

7. George BP, Chandran R, Abrahamse $\mathrm{H}$. Role of phytochemicals in cancer chemoprevention: insights. Antioxidants (Basel) 2021;10:1455.

8. Boreddy SR, Srivastava SK. Pancreatic cancer chemoprevention by phytochemicals. Cancer Lett 2013;334:86-94.

9. Kotecha R, Takami A, Espinoza JL. Dietary phytochemicals and cancer chemoprevention: a review of the clinical evidence. Oncotarget 2016;7:52517-29.

10. Kandhari K, Agraval H, Sharma A, Yadav UCS, Singh RP. Flavonoids and cancer stem cells maintenance and growth. In: Rani V, Yadav UCS, eds. Functional Food and Human Health. Singapore, Springer Singapore, pp 587-622, 2018.

11. Li W, Guo Y, Zhang C, Wu R, Yang AY, Gaspar J, et al. Dietary phytochemicals and cancer chemoprevention: a perspective on oxidative stress, inflammation, and epigenetics. Chem Res Toxicol 2016;29:2071-95.

12. Raina K, Kumar D, Agarwal R. Promise of bitter melon (Momordica charantia) bioactives in cancer prevention and therapy. Semin Cancer Biol 2016;40-41:116-29.

13. Kaur M, Deep G, Jain AK, Raina K, Agarwal C, Wempe MF, et al. Bitter melon juice activates cellular energy sensor AMP-activated protein kinase causing apoptotic death of human pancreatic carcinoma cells. Carcinogenesis 2013;34:1585-92.

14. Dhar D, Deep G, Kumar S, Wempe MF, Raina K, Agarwal C, et al. Bitter melon juice exerts its efficacy against pancreatic cancer via targeting both bulk and cancer stem cells. Mol Carcinog 2018;57:1166-80.

15. Lucas EA, Dumancas GG, Smith BJ, Clarke SL, Arjmandi BH. Health benefits of bitter melon (Momordica charantia). In: Watson RR, Preedy VR, eds. Bioactive Foods in Promoting Health: Fruits and Vegetables. San Diego, Academic Press, pp 525-49, 2010.

16. Nerurkar PV, Lee YK, Motosue M, Adeli K, Nerurkar VR. Momordica charantia (bitter melon) reduces plasma apolipoprotein B-100 and increases hepatic insulin receptor substrate and phosphoinositide-3 kinase interactions. Br J Nutr 2008;100:751-9.

17. Dandawate PR, Subramaniam D, Padhye SB, Anant S. Bitter melon: a panacea for inflammation and cancer. Chin J Nat Med 2016;14:81-100.

18. Reed D, Kumar D, Kumar S, Raina K, Punia R, Kant R, et al. Transcriptome and metabolome changes induced by bitter melon (Momordica charantia)- intake in a high-fat diet induced obesity model [published online ahead of print August 19, 2021]. J Tradit Complement Med. doi: 10.1016/j.jtcme.2021.08.011.

19. Kavitha CV, Agarwal C, Agarwal R, Deep G. Asiatic acid inhibits pro-angiogenic effects of VEGF and human gliomas in endothelial cell culture models. PLoS One 2011;6:e22745.

20. Dhar D, Raina K, Kant R, Wempe MF, Serkova NJ, Agarwal C, et 
al. Bitter melon juice-intake modulates glucose metabolism and lactate efflux in tumors in its efficacy against pancreatic cancer. Carcinogenesis 2019;40:1164-76.

21. da Silva RF, Dhar D, Raina K, Kumar D, Kant R, Cagnon VHA, et al. Nintedanib inhibits growth of human prostate carcinoma cells by modulating both cell cycle and angiogenesis regulators. Sci Rep 2018;8:9540.

22. Baena Ruiz R, Salinas Hernández P. Cancer chemoprevention by dietary phytochemicals: Epidemiological evidence. Maturitas 2016;94:13-9.

23. Liu RH. Health benefits of fruit and vegetables are from additive and synergistic combinations of phytochemicals. Am J Clin Nutr 2003;78(3 Suppl):517S-20S.

24. Agraval H, Yadav UCS. MMP-2 and MMP-9 mediate cigarette smoke extract-induced epithelial-mesenchymal transition in airway epithelial cells via EGFR/Akt/GSK3 $\beta / \beta$-catenin pathway: amelioration by fisetin. Chem Biol Interact 2019;314:108846.
25. Shostak A. Circadian clock, cell division, and cancer: from molecules to organism. Int J Mol Sci 2017; 18:873.

26. Hanahan D, Weinberg RA. Hallmarks of cancer: the next generation. Cell 2011;144:646-74.

27. Leung L, Birtwhistle R, Kotecha J, Hannah S, Cuthbertson S. Anti-diabetic and hypoglycaemic effects of Momordica charantia (bitter melon): a mini review. Br J Nutr 2009;102:1703-8.

28. Fuangchan A, Sonthisombat $P$, Seubnukarn $T$, Chanouan $R$, Chotchaisuwat $P$, Sirigulsatien $V$, et al. Hypoglycemic effect of bitter melon compared with metformin in newly diagnosed type 2 diabetes patients. J Ethnopharmacol 2011;134:422-8.

29. Alam MA, Uddin R, Subhan N, Rahman MM, Jain P, Reza $\mathrm{HM}$. Beneficial role of bitter melon supplementation in obesity and related complications in metabolic syndrome. J Lipids 2015;2015:496169.

30. Nikitenko L, Boshoff C. Endothelial cells and cancer. Handb Exp Pharmacol 2006;(176 Pt 2):307-34 